\title{
Echoes of Photons Past
}

High Harmonic Generation is an established method to significantly upshift laser photon energies. Now, researchers at the SLAC National Accelerator Laboratory have used echo concepts to generate coherent high-harmonic output from an electron beam light source.

Lawrence T. Campbell and Brian W.J. McNeil

Significant research has been carried out worldwide towards upshifting the photon energy of conventional laser output. An example is High Harmonic Generation ${ }^{1}$ (HHG) which uses high power IR drive lasers to generate output into the EUV (a few 10's of $n$ m). However, output powers from HHG tail off toward the water window at wavelengths of 2-4nm, where there are a great number of applications. HHG sources can be amplified to much higher powers using a Free Electron Laser ${ }^{2,3}$ (FEL), but the power produced by HHG sources in the water window is currently insufficient to properly seed the FEL interaction. On page XXX of this issue ${ }^{4}$, Erik Hemsing and colleagues describe an experiment at the SLAC National Accelerator Laboratory which demonstrates the direct generation of high harmonics from an infra-red (IR) laser driven electron beam using a method called Echo-Enabled Harmonic Generation (EEHG). In the future, it should be possible to replace the IR drive lasers with shorter wavelength HHG sources so that EEHG could provide high power coherent sources into the water window.

High gain amplifier FELs operate via a collective, positive feedback interaction between a copropagating beam of relativistic electrons and the resonant light they emit in an undulator ${ }^{3}$. The electrons form a density grating by 'bunching' at the resonant wavelength to emit coherently. Without a coherent seed field of sufficient power, the FEL starts up from the spontaneous radiation emission arising from the intrinsic shot noise of the electron beam and the electron bunching and radiation evolve to give a saturated output of poor temporal coherence. If the FEL is seeded using a laser with sufficient power, it retains the good coherence properties of the initial seed through to final saturated output. Unfortunately, there are no seed sources with sufficient power to coherently seed an FEL in the water window.

In EEHG however, the electron beam is manipulated using drive lasers and dispersive magnetic chicanes to carefully pre-bunch the electrons at high harmonics of the drive lasers before any emission takes place. The harmonically bunched beam retains the good coherence properties of the drive lasers. When injected into an undulator tuned to the pre-bunched beam, the electrons immediately radiate coherently at powers above the noisy, spontaneous power. This coherent seeding can be maintained and subsequently amplified through to saturation. Like any good recipe, the secret of success lies in the preparation.

Electron beam pre-preparation in the EEHG method consists of two main stages. In the first stage, a laser and undulator sinusoidally modulate the electron beam energy so that the electrons begin to disperse, with the higher energy electrons moving ahead and the lower energy falling behind. A strong magnetic chicane enhances this dispersive process, and shears the modulated electrons along their direction of motion. Imagine taking all the peaks and troughs of the energy modulated beam and stretching them many modulation periods in opposite directions along the propagation direction. The beam is then composed of many layers of 'beamlets' each of a different energy - but still no bunching. The second stage then energy modulates this layered beam again, usually at a longer wavelength, to form a beam composed of many sinusoidally modulated beamlets, each of slightly different mean energy and stacked one on top of each other. A second, much weaker 
dispersive magnetic chicane then simultaneously shifts each energy modulated beamlet according to its mean energy and disperses the beamlets' energy modulation to form a density grating. Not only is the beam now bunched, but the bunching period is at a much smaller, high harmonic wavelength of the second modulating laser. The bunched beam also retains the good temporal coherence properties of the second modulating laser and is now ready to emit coherently.

Other methods have also been proposed and tested to seed an FEL interaction using a longer wavelength conventional laser with the aim of performing harmonic up-conversion while retaining the good temporal coherence properties of the seed. One is High Gain Harmonic Generation ${ }^{5}$ (HGHG), a process which may be cascaded to obtain larger harmonic upshifts ${ }^{6}$. However, as its name suggests, this process relies upon the high-gain FEL mechanism so that, unlike EEHG, the effects of intrinsic noise or beam inhomogeneities can become amplified during the harmonic up-shifting process, limiting the total harmonic upshift achievable ${ }^{7,8}$. This appears to give the edge to methods like EEHG which pre-prepare the electron beam bunching before emission, so reducing amplification of unwanted noise.

In their experiments the SLAC team used a scaled-down version of a water window EEHG source, using a second modulation laser wavelength of $2400 \mathrm{~nm}$ to generate coherent output in the radiating undulator down to $32 \mathrm{~nm}$, the maximum harmonic up-shift of 75 that was obtained and an impressive five-fold increase over previous EEHG experiments. It is interesting to note that the electron beam preparation in the two modulation-chicane modules was carried out at a beam energy of $120 \mathrm{MeV}$. The highest harmonic up-shifts were then achieved by further accelerating the high harmonic bunched electron beam in a short RF linac up to an energy of $192 \mathrm{MeV}$ before it was injected into the radiating undulator. Typical values for a water window EEHG source using this harmonic up-shift would be modulation wavelengths in the UV $(\sim 200 \mathrm{~nm})$, for example using HHG sources as the drive lasers, and electron beam energies of $\sim 1-2 \mathrm{GeV}$.

One impressive element to the results reported is the close agreement of the theoretical models to the experiment. When discussing classical systems that upshift frequencies by the large factors discussed, one might expect some discrepancies to creep in. However, the agreement of the theory to the experimental results is excellent. For example, the theory predicts that specific high harmonics may be enhanced or suppressed with respect to their neighbours by fine tuning of the final chicane and exactly this behaviour is observed. This detail gives great comfort that the predictions of water window performance are well based.

While impressive harmonic up-shifts were obtained, the experiments only generated coherent output powers at the shortest wavelengths of a few times the spontaneous output that occurs in the absence of any electron bunching. This reduced output is attributed to the electron beam transport through the radiating undulator. The electron beam needs a distributed system of focussing magnets to maintain a constant beam radius, otherwise the beam spreads out - a bit like free space diffraction of a light beam. However, the focusing system introduces a 'smearing' of the electron bunching as it propagates through the focussing, and this in turn reduces coherent emission. The good news is that scaling up to the higher energy beams needed for a water window EEHG system, these effects are greatly reduced. Estimates of seed power output in the water window are at the around 4 orders of magnitude above the incoherent spontaneous power allowing FEL amplification potentially above GW power levels. This would be a powerful source of coherent radiation in a very important region of the spectrum. 
The SLAC experiment certainly paves the way towards a powerful, coherent water window EEHG source. Recent proposals take this a step further by introducing a two-beam EEHG approach to reach the hard X-ray ${ }^{9}$. We think we will be hearing a lot more of this method in issues to come.

\footnotetext{
${ }^{1}$ Tenio Popmintchev, Ming-Chang Chen, Paul Arpin, Margaret M. Murnane \& Henry C. Kapteyn, The attosecond nonlinear optics of bright coherent X-ray generation, Nature Photonics 4, 822-832 (2010)

${ }^{2} \mathrm{G}$. Lambert et al, Injection of harmonics generated in gas in a free-electron laser providing intense and coherent extreme-ultraviolet light, Nature Physics 4, 296 - 300 (2008)

${ }^{3}$ Brian W. J. McNeil and Neil R. Thompson, X-ray free-electron lasers, Nature Photonics 4, 814-821 (2010)

${ }^{4}$ E. Hemsing et al, Ultra high echo-enabled harmonics from precisely tailored electron beams, Nature Photonics XXX, XXX - XXX (2016)

${ }^{5}$ L. H. Yu, Generation of intense UV radiation by subharmonically seeded single-pass free-electron lasers, Phys. Rev. A 44, 5178-5193 (1991)

${ }^{6}$ E. Allaria et al, Two-stage seeded soft-X-ray free-electron laser, Nature Photonics 7, 913-918 (2013)

${ }^{7}$ E.L. Saldin, E.A. Schneidmiller and M.V. Yurkov, Study of a noise degradation of amplification process in a multistage HGHG FEL, Optics Communications 202, 169-187 (2002)

${ }^{8} \mathrm{G}$. Penn, Stable, coherent free-electron laser pulses using echo-enabled harmonic generation, Phys. Rev. ST$A B, 17,110707$ (2014)

${ }^{9}$ Zhentang Zhao, Chao Feng, Jianhui Chen and Zhen Wang, Two-beam based two-stage EEHG-FEL for coherent hard X-ray generation, Science Bulletin 61, 720-727 (2016)
} 